\title{
Modeling of Water Vapor Condensation by Using Computer Methodology
}

\author{
Jan Skramlik, Miloslav Novotny and Karel Suhajda \\ Department of Building Constructions, Faculty of Civil Engineering, Brno University of Technology, Brno 602 00, Czech Republic
}

\begin{abstract}
The excessive moisture has an adverse effect on the building materials structure. Most standard construction materials are characterized by porous structure, resulting in the ability to absorb water in liquid and gaseous phases in the inner pores. Under certain conditions, water fills the pores within the structure of building material and then moves back to its surrounding. Many technical studies have shown that monitoring the moisture transport is mainly based on experimental methods. This work is based on models of transport of moisture in building physics, i.e., the description of the moisture behaviour of building materials based on physical laws models (KRISCHER, KIESSL). The aim of this work is to obtain the parameters of distribution of moisture for calculation capillary conductivity coefficient for practical using by means of non-destructive method. The authors have now developed all the software required to perform a boundary element analysis of problems in potential flow. The examples which the authors can analyse will, however, be restricted to homogenous domains.
\end{abstract}

Key words: Capillary conductivity coefficient, moisture transfer, EMWR (electromagnetic microwave radiation), diffusion.

\section{Introduction}

Excessive moisture in building materials affects the physical characteristics of building structures and causes its degradation. Apart from a few exceptions, building materials always contain moisture. To evaluate the negative effects of humidity on the material, which is used in building construction, it is necessary to determine its moisture characteristics. Every building construction that forms the building envelope and is made of porous materials should be designed with respect to a number of factors that somehow impede its function. Such factors include water vapor - as a liquid moisture phase. Diffusion of water vapor is sufficiently explored but regarding moisture transport in porous structure there is in some circumstances water vapor involved in its liquid phase. Therefore, it should be considered decisive for the transport of moisture. However, it is necessary to know information about its expansion in porous media and in

Corresponding author: Jan Skramlik, Ph.D., associate professor, research field: building construction. E-mail: skramlik.j@fce.vutbr.cz. particular about the condensation of water vapor. If water vapor condensed in porous material, liquid moisture emerges in part of construction. If there is a liquid moisture gradient in a porous material, it is an indicator of liquid moisture moving through in capillaries. This phenomenon is called capillary conductivity of moisture.

\section{Description of Moisture Distribution}

Water gets in porous material from the environment and the equilibrium between the material moisture and the ambient air moisture is reached under steady temperature and moisture conditions. If the partial pressure of water vapor in a building material is lower than that in the ambient air, then the material absorbs water vapor from the air. An impact of moisture on coefficients of thermal conductivity varies according to the real temperature and moisture content of a material. Material moisture is characterized by water content in the pores of a material and is expressed by the weight or volume parts of water in proportion to the solid phase of a material. Moisture diffusion processes are 
accelerated by water vapor and with greater temperature gradient the expansion also increases.

\subsection{Diffusion in Capillary Medium}

Porous materials consist of cavities in various forms. Many technical studies show that the moisture monitoring is prevailingly based on experiments. Previous calculating methods introduced, e.g., by Glaser, which became the basis for the standard calculations in many European countries in the 1960's are not always sufficient with respect to the demands for the civil structures.

Fig. 1 shows a cross section of the capillary with prerequisite of moisture transport.

Fig. 2 shows the moisture layer in environment of capillary under influence of condensation of water vapor. Diffusion resistance is lower than in humid conditions. The increase of water vapor permeability occurs at isothermal conditions.

\subsection{Moisture Accumulation in Porous Material}

Sorption and desorption isotherm expresses the value of the absorption and release of moisture from the air at different temperature different relative humidity and the state when air reaches equilibrium moisture. When the relative humidity in porous materials is just below $100 \%$ then sorption isotherm rises steeply which results in capillary condensation (fine pores quickly absorb water).

Progressive increase in material moisture from $60 \%$ relative humidity is attributed to capillary condensation and this makes change of the sorption curve (Fig. 3) [1].
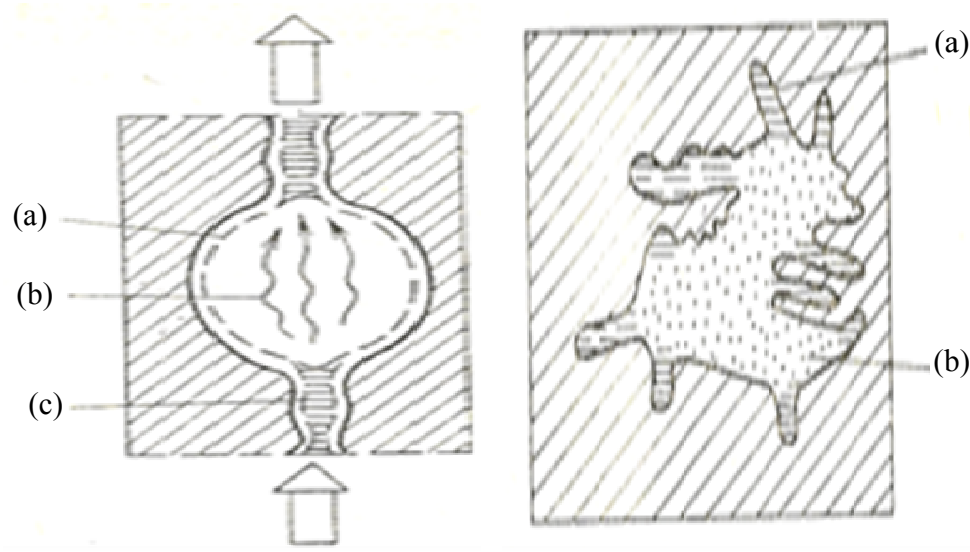

Fig. 1 Example of capillary profile: (a) water; (b) water vapour diffusion; (c) adhesive water.

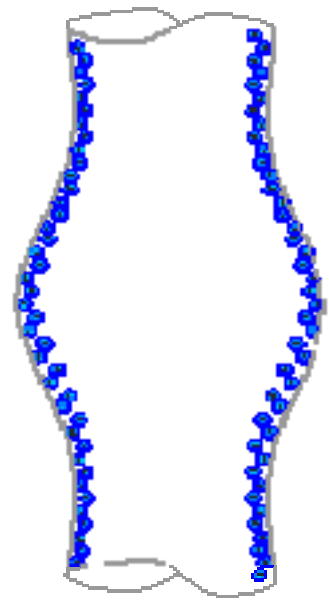

Fig. 2 Monolayer and multilayer moisture in porous.

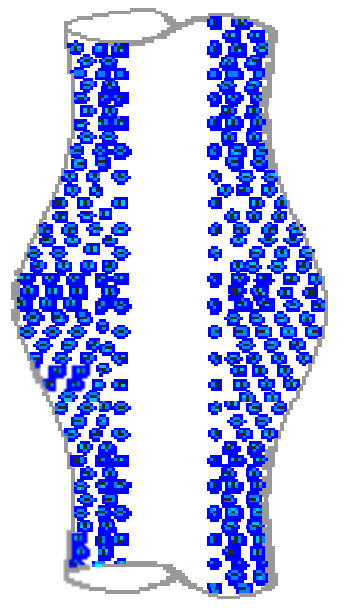
coefficient.

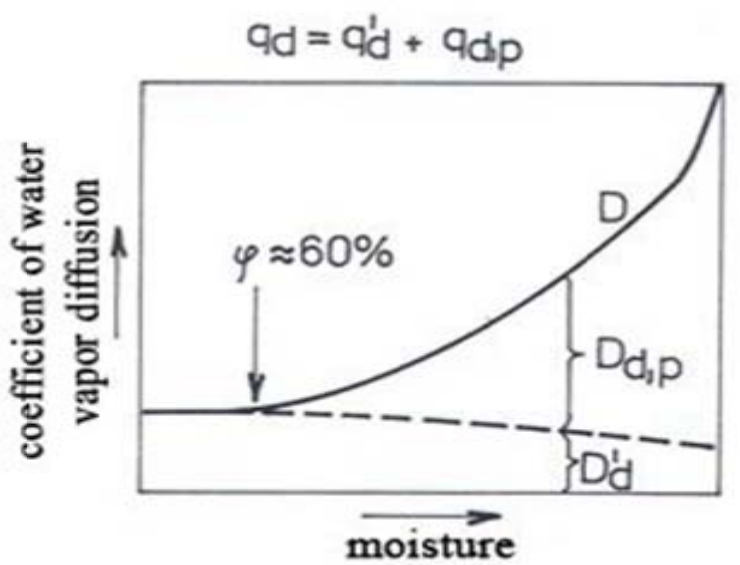

Fig. 3 Dependence of dampness on the diffusion 

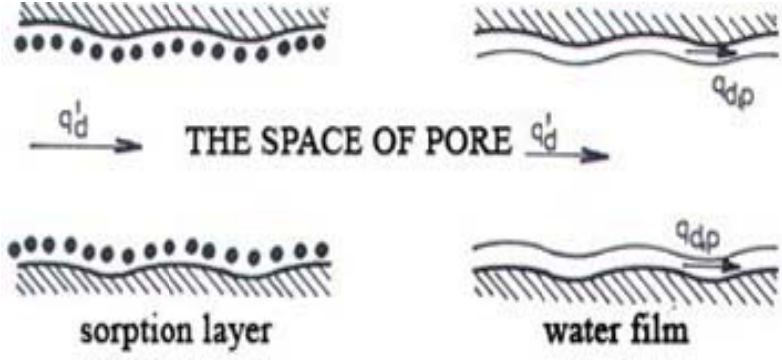

Fig. 4 Distribution of moisture in porous space.

As is shown in Fig. 4, higher dampness $q_{d p}$ supports the spread moisture $q_{d}{ }^{\prime}$. On the surface of capillaries is gradually created water film and a flow of moisture [2].

\subsection{Condensation Zone}

The condensation occurs between the points A and B as seen on the graph (Fig. 3). If the diffusion flow density value at the beginning of the condensation process is $q_{d A}$ and the diffusion flow density value at the end of the process is $q_{d B}$, the amount of condensed water vapor may be calculated as follows (Fig. 5) [3]:

$$
q_{d}=q_{d A}-q_{d B}
$$

In reality dampness does not remain in delimited zone. Condensation extends in porous material in all directions and is affected by moisture gradient and thermal changes. Dampness spreads because of porous texture of building material in a wider range then it is described according to Glaser model-depending also on outer conditions - for example within the range of $A_{x}-B_{x}$ (Figs. 6 and 7). This fact subsequently effects the physical characteristics of building materials and also changes heat conductivity $\lambda$ of material.

\subsection{Waveform of Moistening}

In Fig. 8, there is presented humidity profile $u(x)$ at time intervals $t_{x}$ while moistening porous material with marked initial and boundary conditions for determining the coefficient of capillary conductivity. $A$ is the starting coordinate of the profile of the wetting front, $B$ is the length of the wet section, and $C$ is the dimension into the dry region $\rightarrow \infty$. Along the vertical axis are presented the values of moisture weight, where $u_{2}$ is moisture weight in the measured sample and $u_{1}$ is moisture weight in the measured sample after its moistening. By combining the Luikov equation, which describes the transport of moisture within porous materials, and the continuity equation, then the authors get equation in form as follows:

$$
q=-\rho_{S} \kappa \frac{d u}{d x}
$$

In Fig. 5, $R$ is diffusion resistance, $p(P a)$ is pressure and $\tau\left({ }^{\circ} \mathrm{C}\right)$ is temperature.

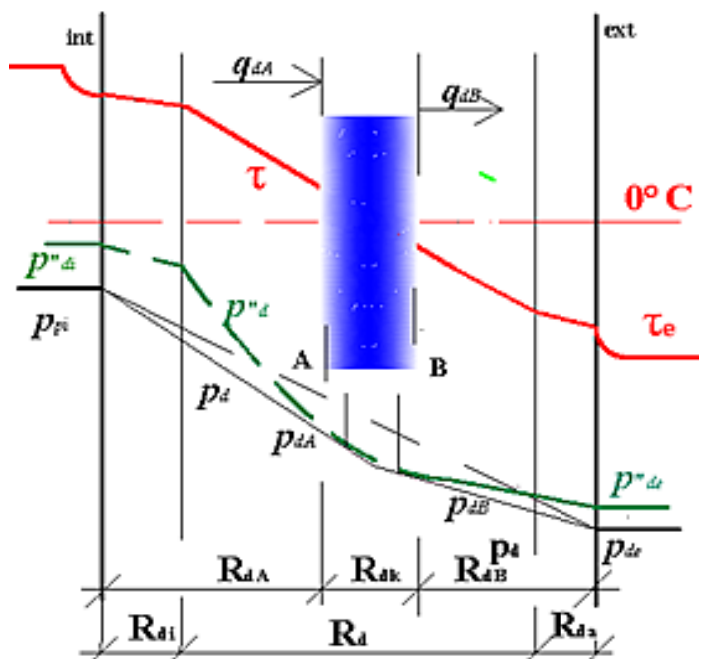

Fig. 5 Determination of the condensation zone.

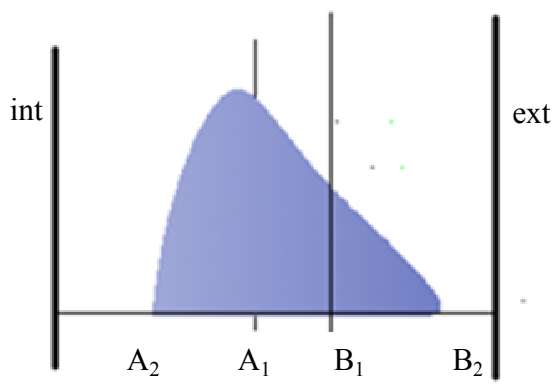

Fig. 6 Condensation outside of condensation zone.

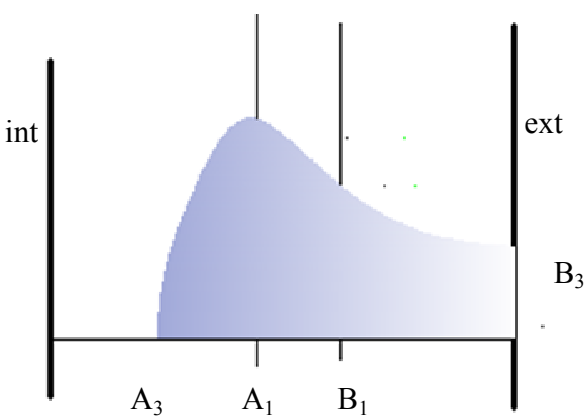

Fig. 7 Transport of condensate to the surface. 


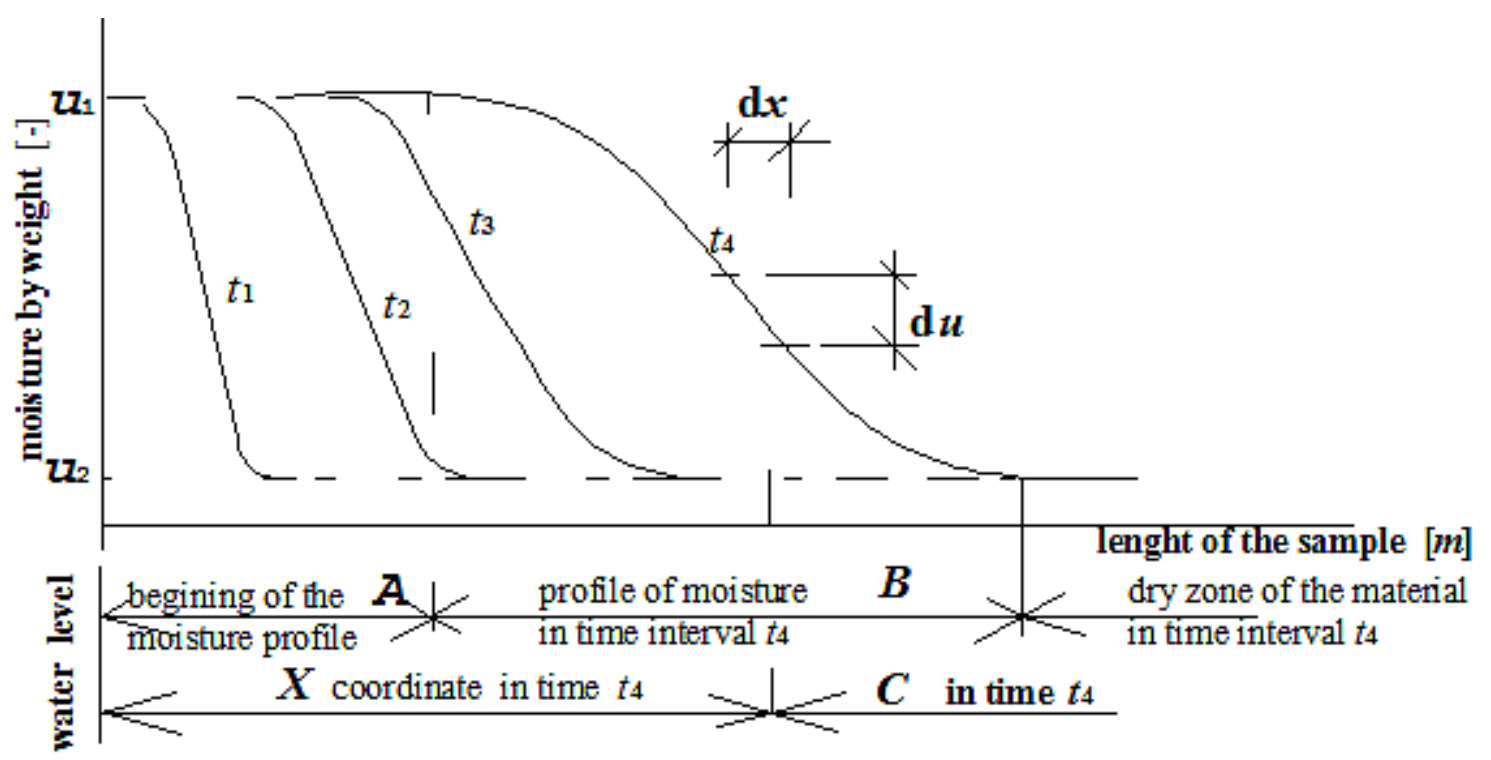

Fig. 8 Moisture distribution $u(x)$ at various time intervals $t_{x}$.

$$
\rho_{S} \cdot \frac{\partial u}{\partial t}=\nabla \cdot q=-\operatorname{div} q
$$

If the measurements is done in such a way that the liquid can rise through the porous material only in the direction of the $x$ axis, and the humidity gradients are equal to zero, then the last equation can be expressed in one-dimensional form [3]:

$$
\rho_{S} \cdot \frac{\partial u}{\partial t}=-\nabla \cdot\left(-\rho_{S} \cdot \kappa \cdot \nabla u\right)
$$

after adjustment:

$$
\frac{\partial u}{\partial t}=\frac{\partial}{\partial x}\left(\kappa \frac{\partial u}{\partial x}\right)
$$

If there is known spatial and temporal distribution of moisture in building material, it is possible to determine the coefficient of capillary conductivity.

\section{Capillary Conductivity Coefficients}

The coefficient of capillary conductivity can be calculated either by method of gradual integration according to the $x$ coordinate $(\xi)$ or by introducing $\xi$ to substitute the distance measured along the samples length from the point on the curve of the wetting front $\mathrm{du}$, expressed in the formula up to $\infty$. In practice, however, it is included in the interval on the measured sample up to the distance, where moisture occurs in steady state, i.e., $u_{2}$, which is the value of relative humidity in the measured material (Fig. 8).

\subsection{Matan Method}

Matan method is the method for determining the coefficient of capillary conductivity, if the authors know one wetting curve and the time since the beginning of the experiment $\dot{\eta}$. To simplify the calculation there is used the function of transformation of two variables on the function of one variable $\dot{\eta}$ [3]:

$$
\eta=\frac{x}{2 \sqrt{t}}
$$

which converts the solution of partial differential equations to solve ordinary differential equations in new variables and after substitution in Eq. (5) is obtained an ordinary differential equation [3]:

$$
\frac{\partial}{\partial \eta} \cdot\left(k(u) \cdot \frac{\partial u}{\partial \eta}\right)+2 \eta \frac{\partial u}{\partial \eta}=0
$$

from where:

$$
\kappa(u) \cdot u^{\prime}(x) \cdot 2 \sqrt{t}=\int_{x(u 2)}^{x(u 1)}-\frac{x}{\sqrt{t}} \cdot u^{\prime}(x) d x
$$

and

$$
\kappa(u(x))=\frac{1}{2 \cdot t \cdot u^{\prime}(x)} \int_{x}^{\infty} \varsigma u^{\prime}(\varsigma) \cdot d \varsigma \quad\left(\mathrm{m}^{2} \cdot \mathrm{s}^{-1}\right)
$$

where,

$\kappa(u(x))$ is coefficient of capillary conductivity $\left(\mathrm{m}^{2} \cdot \mathrm{s}^{-1}\right)$

$t$ is time interval (s);

$\checkmark$ is substitution of the distance measured along the 
sample;

$\eta$ is transformation denoted as Boltzmann coordinate $\left(\mathrm{m} \cdot \mathrm{s}^{-1 / 2}\right)$;

$x$ is coordinate in the length of sample (m);

$u_{l}$ is maximal value of moisture by weight (-);

$u_{2}$ is moisture by weight of material (-).

\subsection{Vapor Transport Coefficient According to ISO Standard}

According to European Standard 15026, the determination of the vapor coefficient is described in EN ISO 12572. In case of high water content the liquid transport has a particular influence on the moisture transport behavior of porous material. The material characteristic to describe this process is the liquid diffusivity or the liquid conductivity. Both transport coefficients are linked by the following equation [4]:

$$
K\left(p_{\text {suc }}\right)=-D_{w}(w) \frac{\partial w}{\partial p_{\text {suc }}}
$$

$D_{w}$ is moisture diffusion as transfer coeff. $\left(\mathrm{m}^{2} \cdot \mathrm{s}^{-1}\right)$;

$p_{s u c}$ is pressure in suction $(\mathrm{Pa})$;

$w$ is moisture by weight $\left(\mathrm{kg} \cdot \mathrm{m}^{-3}\right)$.

Informative mention in conclusion of the European Standard EN ISO 15026 presents the moisture distribution and functional relation between $D_{w}$ and $w_{s}$ on the basis of measurement by NMR (nuclear magnetic resonance) method. The standard presents following data [4]:

$g_{w}=K\left(p_{\text {suc }}\right) \frac{\partial p_{\text {suc }}}{\partial_{x}}=-D_{w}(w) \frac{\partial w}{\partial p_{\text {suc }}} \frac{\partial p_{\text {suc }}}{\partial_{x}}=-D_{w}(w) \frac{\partial w}{\partial x}$

where, $D_{w}(w)$ is as $\kappa(u)$ and according to Krischer [5] the density of liquid water flow rate is defined as follows:

$$
m_{u}=-k_{p} \cdot \rho_{w} \cdot \frac{d p}{d x}
$$

$k_{p}$ is transport coefficient relative to pressure.

\section{Detection of Moisture Using EMWR}

Fig. 9 presents a detail construction of experimentally assembled apparatus for monitoring moisture transport in porous materials. It is made up of a tank to contain liquid, a positioning mechanism, which serves to vary the level of liquid.

The tested sample is placed over the tank with water (Fig. 9) and it is fastened in a stirrup, which is hanging on a digital scale. In the space above the tank is accommodated a waveguide transmitting of microwave radiation, linked to a radiation source on the other side of the tank, opposite of the transmitting waveguide is situated a receiving waveguide. Both waveguides, as one unit, are height adjustable and are arranged on a supporting frame (not shown in the figure).

Fig. 10 shows a sample of the material during measurement.

\subsection{Detection Moisture Distribution}

The values of coordinates determining location of the wetting front profile are obtained from measurements using the microwave measuring

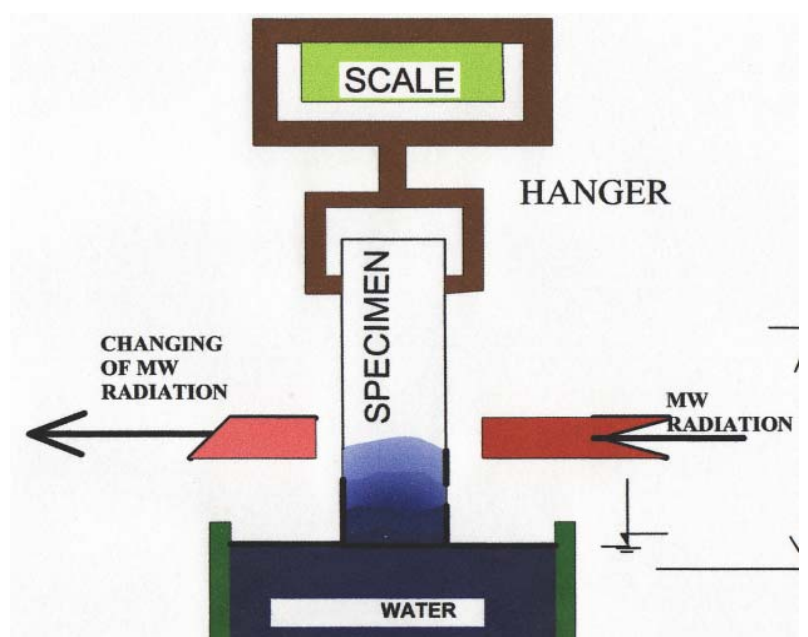

Fig. 9 The principle of saving the test sample material between the waveguides.

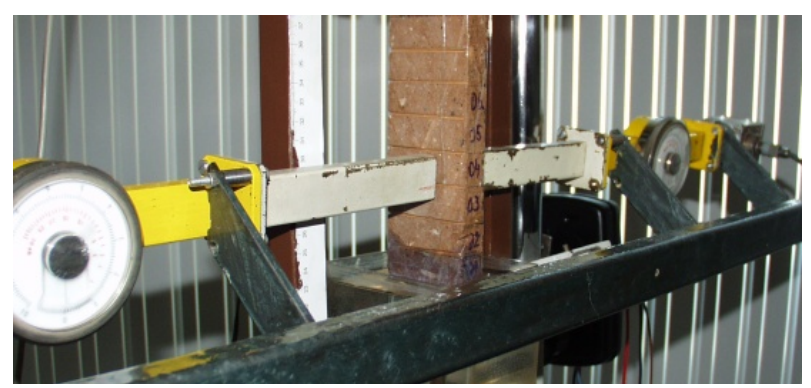

Fig. 10 Detail of the contact sample with water level. 
apparatus. Detection of moisture quantity diffusing in the material is carried out at certain time intervals from the start of moistening of sample material (Section 2.4).

Fig. 11 [6] shows the change of EMWR intensity depending (the $y$ axis) on the moisture by weight in the length of measured sample (the axis $x$ ) at times intervals of $10 \mathrm{~min}$ in the course of its moistening and consequently determines functional dependence on moisture.

Fig. 12 is result of the moisture distribution measurement as the wetting curves using EMWR.

For detecting moisture distribution was assembled individual software that allows record and evaluation of measured data without the human factor.

\subsection{Measurement Methodology and Calculations}

Methodology of measuring using the experimental apparatus was developed on the basis of test measurements on specimens taken from porous that passes through the sample in the distance from the source of moisture.

Dependence of the defined function (Fig. 11):

$$
u_{m, t}=f\left(z_{t}(x)\right)
$$

where,

$u_{m}$ is moisture by weight (-);

$z$ is EMWR which passes through sample (-);

$x$ is coordinates of moisture $(\mathrm{m})$;

$t$ is time interval of measurement (s).

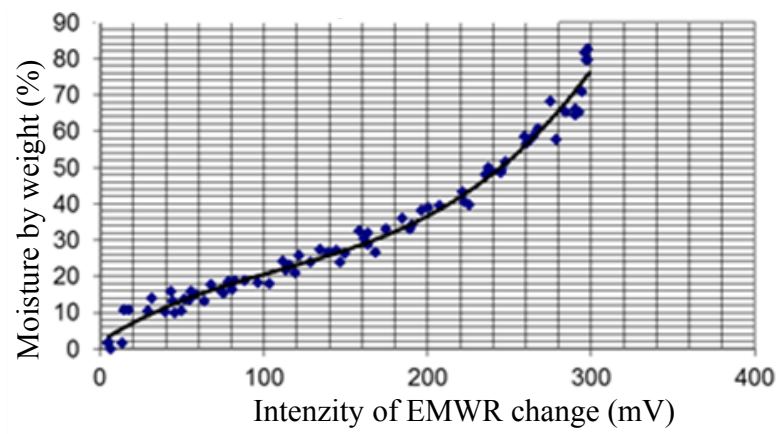

Fig. 11 Functional dependence of EMWR on moisture.

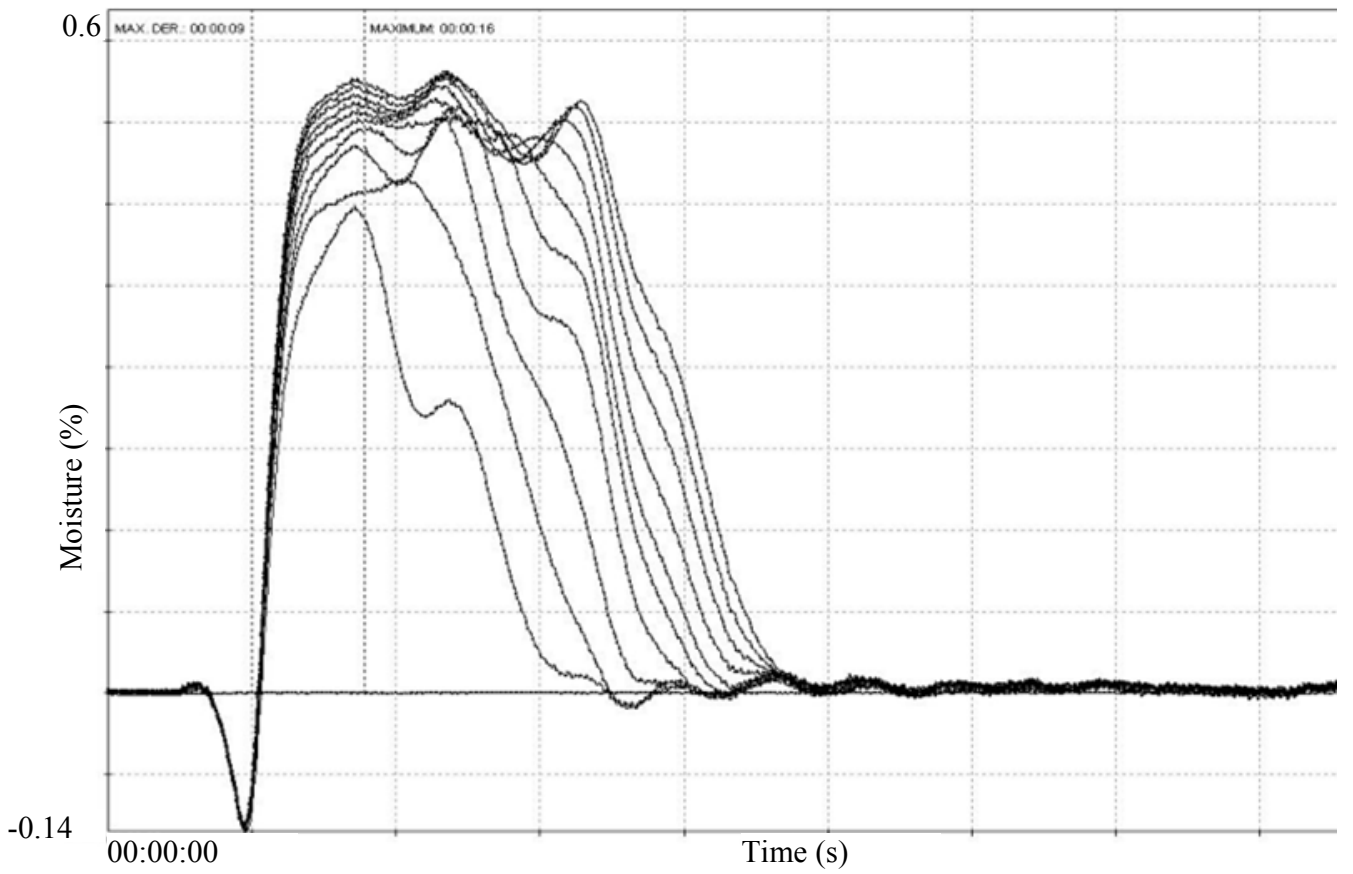

Fig. 12 Measure results into time intervals 10, 20, 30... min. 


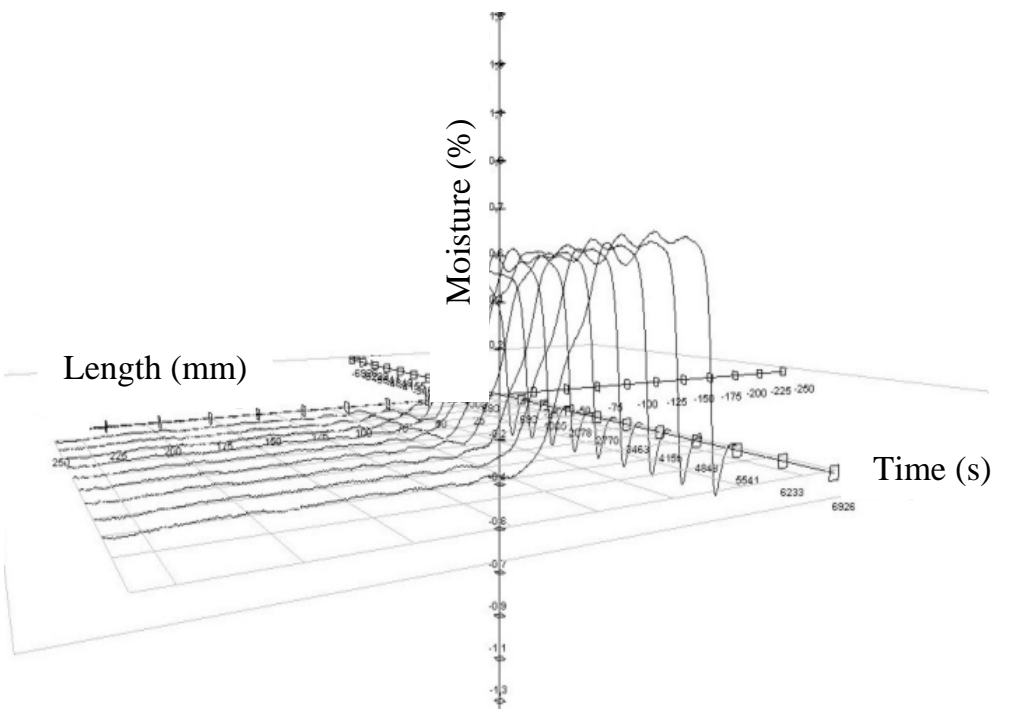

Fig. 13 3D view of the moisture distribution.

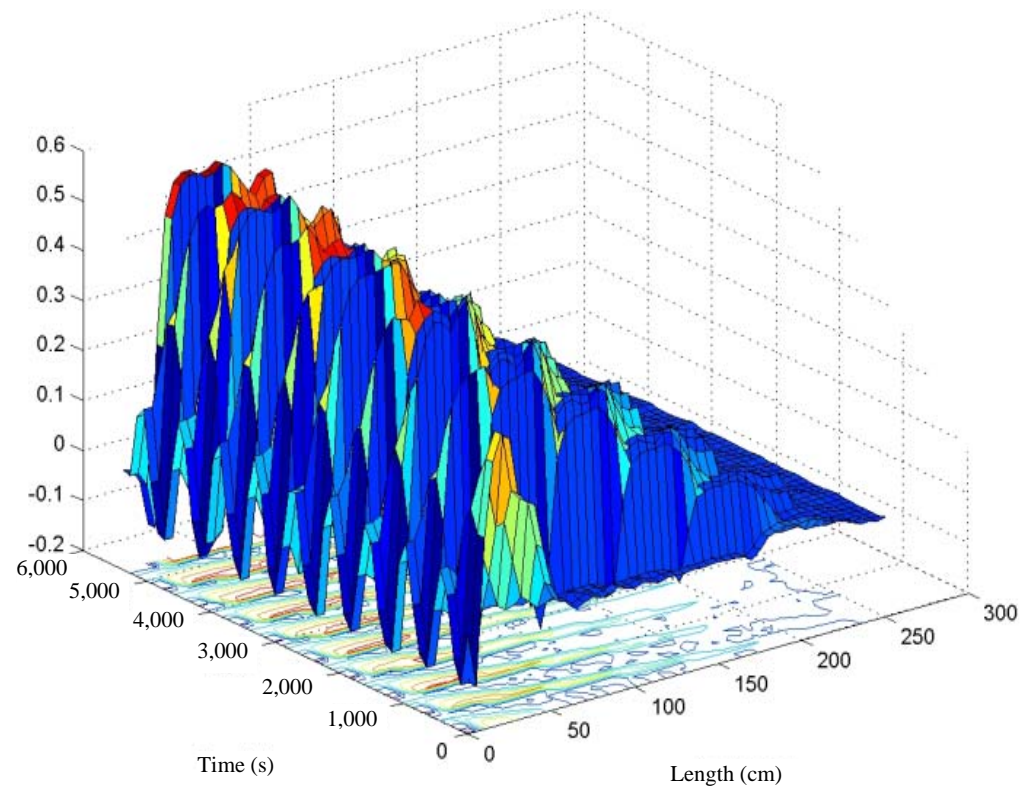

Fig. 14 Data processing in program MATLAB.

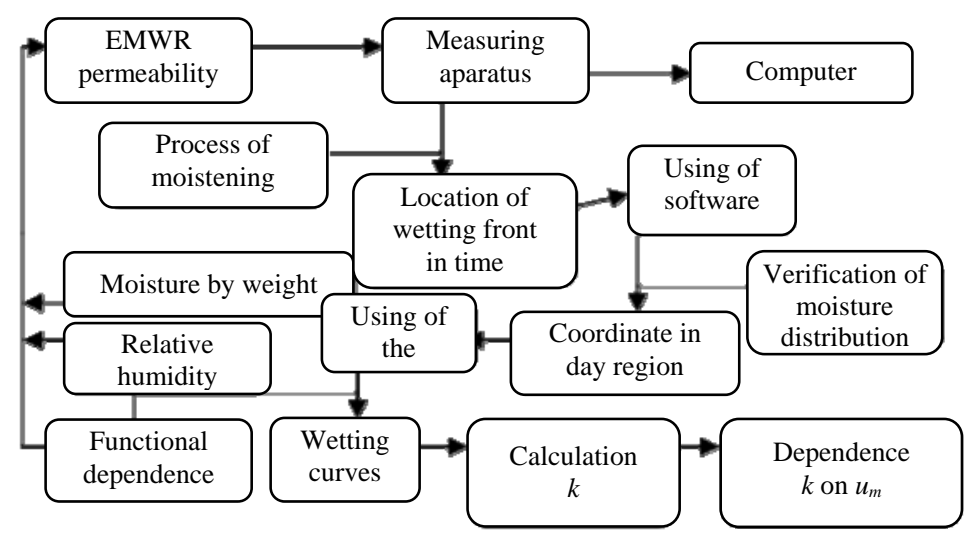

Fig. 15 Procedure for measurement of moisture distribution using EMWR. 


\section{Practical Computations}

Basis for calculating the coefficient of capillary conductivity is the curve of wetting. Fig. 12 shows the output of the measurement using experimentally assembled apparatus.

Measured values of moisture can be displayed spatially in three dimensions - the moisture content depending on the length of the sample material according with time of moistening (Fig. 13).

Fig. 14 shows the measured data of moisture distribution (axe Z) for the computing method to the determination of capillary conductivity coefficient depending on the moisture using, in this case, at computer program MATLAB.

Fig. 15 shows procedure measurement of values to obtain capillary conductivity coefficient and methodology for measurement on the measuring apparatus on principle of using electromagnetic microwave radiation using experimentally assembled apparatus.

To calculate the coefficient of capillary conductivity allows the creation methodology, in comparison with destructive method of obtaining a higher frequency and accuracy of the data moisture conditions in the detailed sections on the length of the reference sample. The advantage is the possibility of continuous measurement of multiple curves wetting a sample of the material in any period of time without interruption measurement.

\section{Conclusions}

Moisture distribution in porous material is currently adequately addressed. The problem remains the condensations of water vapor inside of the porous structures. The aim of this analytical method of modeling of non-destructive measurements of moisture transport is detection diffusion of liquid phase using microwave radiation. Newly is built up measuring device of three-dimensional distribution of moisture. Determination three-dimensional distribution of moisture in the sample material allows accurate determination of moistening curves in optimal times of moistening to using of integral method for determination of the capillary conductivity coefficient.

\section{Acknowledgments}

This article was supported by GACR P104/10/P388 and by GACR P104/10/1390 "the analysis of influence of MW drying while application of chemical grouting into moist brickwork".

\section{References}

[1] H. Künzel, Determination of Moisture by Weight in Relation to the Thermal Conductivity of Materials, Building Physic, Essen, 1986, pp. 33-39.

[2] K. Kiessl, Capillary and vapor moisture transport in building components, Ph.D. Dissertation, University Essen, 1983.

[3] M. Kutilek, Dampness Porous Material, SNTL Publishing, Prague, 1992.

[4] Assessment of Moisture Transfer by Numerical Simulation, European Standard, EN ISO 15026, 2007.

[5] K. Krischer, The Scientific Principles of Drying Technology, Springer Verlag, Germany, 1978.

[6] J. Skramlik, M. Novotny, K. Suhajda, Modelling of diffusion in porous medium, ICNAAM Khalkidhiki, American Institute of Physics 1389 (2011) 1869-1873. 